\title{
STAGES IN THE EVOLUTION AND DEVELOPMENT OF SIGN USE (SEDSU)
}

\author{
JORDAN ZLATEV \\ Lund University, Department of Languages and Literature, \\ Box 201, 22100 Lund, Sweden
}

\author{
THE SEDSU PROJECT ${ }^{*}$
}

Centre for Cognition, Computation and Culture, Department of Psychology, Goldsmiths, University of London London, SE14 6NW, UK

\begin{abstract}
We present the rationale and ongoing research of an interdisciplinary international project aiming at developing a novel theory of semiotic development, on the basis of broad developmental, cross-species and cross-cultural research. We focus on five socialcognitive domains: (i) perception and categorization, (ii) iconcity and pictures, (iii) space and metaphor, (iv) imitation and mimesis and (v) intersubjectivity and conventions, each of which is briefly described. Our main hypothesis is that what distinguishes human beings from other animals is an advanced capacity to engage in sign use, which on its part allowed for the evolution of language.
\end{abstract}

\section{Introduction}

There is no consensus about what makes humans intellectually and culturally different from other species, and even less so concerning the underlying sources of these differences. The main hypothesis of the project Stages in the Evolution and Development of Sign Use (SEDSU) is that it is not language per se, but an advanced ability to engage in sign use that constitutes the characteristic feature of human beings. In particular, this implies the ability to differentiate between the sign itself, be it gesture, picture, word or abstract symbol, and what it represents, i.e. the sign function (Piaget, 1945), and thus to use (the same) sign systems for both communication and cognition. The SEDSU project is highly interdisciplinary, involving developmental and cognitive psychologists, linguists, philosophers, primatologists, and semioticians from five European countries and Brazil, and fieldwork in Europe, South America, Africa and Asia. This single research effort affords new possibilities for methodological

\footnotetext{
* Ingar Brinck (Lund University), Josep Call (MPI-EVA Lepizig, Partner Leader), Jules Davidoff (Goldsmiths, Project Coordinator), Christine Deruelle (INCM-CNRS Marseille), Joël Fagot (INCM-CNRS Marseille, Partner Leader), Peter Gärdenfors (Lund University), Pam Heaton (Goldsmiths), Stephen Nugent (Goldsmiths), Patrizia Poti (ISTC-CNR Rome), Vasu Reddy (Univesity of Portsmouth), Wany Sampaio (Federal University of Rondônia) Chris Sinha (University of Portsmouth, Partner Leader), Göran Sonesson (Lund University), Giovanna Spinozzi (ISTC-CNR Rome, Partner Leader), Elisabetta Visalberghi (ISTC-CNR Rome), Jörg Zinken (University of Portsmouth)
} 
innovation, and the collection and analysis of developmental, cross-cultural and cross-species data in a joint theoretical framework.

Our central research objective is to investigate the developmental and comparative distribution of semiotic processes and their effect on cognition. For this purpose we have singled out five social-cognitive domains and study their interrelations and role in the development of sign use (see Section 2). These domains are all characterised by stage-like developmental profiles that correlate with differences in sign use. The investigations in the different domains are being carried out in parallel, with extensive sharing of methodologies and results. Our ultimate goal is to integrate all the results of the SEDSU project in a coherent new theory of semiotic development, placing the question of the evolution of language in a broader perspective. In this article, we outline our general theoretical orientation, describe some of our ongoing work in each of the five social-cognitive domains, and outline how it contributes to an integrated theory of semiotic evolution and development.

\section{Sign use and the five social-cognitive domains}

Research in the last decades has established significant continuities between humans and non-human species, particularly primates. Nevertheless, when it comes to determining what makes humans unique, it is often claimed that there is one ability - language - that makes human beings special (Christiansen \& Kirby, 2003). However, it could be argued that there are more basic differences between our species and others; for example, representational activity (Piaget, 1945), mimesis (Donald, 1991), and understanding (communicative) intentions (Tomasello, 1999). We would suggest that all these proposals crucially involve differential abilities in sign use. Taking a semiotic perspective and distinguishing between different types of sign systems on the basis of factors such as expression-meaning relation (icon/index/symbol), intentionality, conventionality and complexity permits a gradient approach. This enables us to characterise their emergence in terms of stages, allowing us to situate discontinuities between human and non-human cognition and communication within a broadly continuous evolutionary-developmental framework. Furthermore, studying sign use allows us to scrutinise the semiotic capacities of other species, pre-linguistic and impaired children.

In the SEDSU project we investigate a number of social-cognitive domains characterised by stage-like profiles, where some transitions are more quantitative, while others appear to be qualitative. The domains are: perception and categorisation, iconicity and pictures, space and metaphor, imitation and mimesis and intersubjectivity and conventions. While these may be studied separately, we would argue that they interact so closely in both evolution and ontogeny, that an integrative approach is required. In order to provide an account of the link from individual attention to joint linguistic reference we 
must inquire into the differences between perceptual and linguistic discrimination, the role of pictures as signs, the conceptualisation of space, the relation between imperative and declarative pointing and the role of bodily mimesis.

\subsection{Perception and categorization}

In studying this domain, we consider the possible reorganization of information around a focus of attention as a function of sign use. In order to visually identify objects and segregate them from the background, organisms must be able to group their component parts into perceptual wholes. Comparative studies, however, point to important differences between humans and non-human primates. For example, faced with hierarchical stimuli, several primate species, such as tufted capuchins (Spinozzi, De Lillo \& Truppa, 2003) and chimpanzees (Fagot \& Tomonaga, 1999) process the local details better than the global structure. These findings contrast sharply with the well-known phenomenon of "global advantage" showed by humans. Our hypothesis is that this difference relates to sign use in general, and linguistic performance in particular.

Recent cross-linguistic and phylogenetic investigations (Davidoff, Davies \& Roberson, 1999; Fagot, Goldstein, Davidoff \& Pickering, in press) have also shown a linguistic basis to performance on what again might appear to be solely perceptually based tasks. These studies have indicated that cultural and linguistic training “distorts” perception by stretching perceptual distances at category boundaries. Such effects that depend on both discrimination between categories and identification within category boundaries allow objects to be recruited for sign use by labelling (Brinck, 2003). To further scrutinise the interaction between perceptual processing and sign use we are exploring phylogenetic and developmental trends in perceptual categorisation tasks. These studies were designed so that they could be comparatively conducted in nonhuman primates and in different groups of children (normal, autistic and deaf).

The question remains whether global categorization has been selected for in primate and hominid evolution and can account for some of the difficulties that children with autism encounter with language acquisition. Our preliminary results show a complicated pattern with respect to our target populations. The Marseille group focussing on visual stimuli, have shown that children with autism show a local, as opposed to global, processing bias, which is also the case for baboons. Chimpanzees, in contrast show some intermediary performance. The Goldsmiths group have collected new evidence for enhanced local colour memory in cognitively impaired children with autism. However, they have shown that, while autistic children exhibit a local bias, this does not prevent normal global processing within the musical domain (Heaton, in press). To complicate matters further, there is tentative evidence that the Himba from 
Namibia also have a local processing bias in the visual domain. So, it remains to be shown how categorization might vary under these processing differences.

\subsection{Iconicity and pictures}

According to classical semiotic theory (Peirce, 1931-58) icons are signs that resemble the thing for which they stand, indices are signs that are connected to their referent by means of some independently known or perceived relationship; symbols, on the other hand, are conventional. It has therefore often been argued that icons and indices are elementary phenomena, common to most animals, while symbols are unique to the human species. In order to grasp the similarities and differences in the sign use of human beings, other species, children and individuals suffering from disorders of the semiotic capacity, we separate the properties of iconicity, indexicality, and symbolicity per se from the sign function, defined by Piaget (1945) in terms of differentiation between expression and content. Iconicity and indexicality could conceivably be simple properties accessible to many animals, giving rise to the perception of sameness and/or category membership, and S-R relations, respectively. In contrast, the use of iconic signs such as pictures appears to be a highly sophisticated capacity only found in humans and perhaps some higher primates.

A picture is a surface equipped with markings giving rise to a vicarious perception of objects and actions of the perceptual world (Gibson, 1982). In order to see a picture as a picture, i.e., as a sign, it is necessary to perceive at the same time the similarity and the difference between the surface and that which it depicts; this, according to Gibson, is a capacity only found in human beings. In order to investigate Gibson's surmise, we distinguish primary iconical signs in which the perception of similarity precedes the knowledge of a sign relationship between picture and depicted, and secondary iconical signs, in which the opposite is the case. Primary iconical signs such as pictures seem to presuppose a distinction between two-dimensionality and three-dimensionality (Sonesson, 2000), which has independently been shown to be difficult to grasp for at least some non-human primates (Barbet \& Fagot, 2002). Donald (1991) has suggested that picture use follows language and requires the ability to handle organism-independent representations, which originate with pictures but at later stages render possible writing and theoretical thinking. If so, language may conceivably be a necessary, but not a sufficient, condition for the development of organism-independent representations such as pictures. However, this view is contradicted by experimental investigation of picture use in non-human primates, suggesting that differentiation is possible at least in enculturated chimpanzees. We are currently conducting experiments attempting to show picture-as-sign understanding in (non-enculturated) baboons and chimpanzees. 


\subsection{Space and metaphor}

The spatial domain has been central to recent research into the origins of symbolization, the cognitive foundations of language, and the motivation of linguistic conceptualisation by both universal and culturally specific cognitive processes. Landmarks are perceptible environmental elements or objects that can be used to locate hidden goals. It has been suggested that appreciating the spatial-designation function of landmarks indicates achieving a "symbolic" understanding and that practical achievements in the domain of spatial cognition such as using landmarks could be a pre-requisite for identifying spatial relations in language. Since nonhuman primates use landmarks to locate objects in space (e.g., Potì, Bartolommei, Saporiti, 2005), we are assessing to what extent this use is based on different cognitive processes or on different levels of the same process as in humans, which would also have implications for the relations between spatial language and spatial cognition in humans.

It has been proposed that properties of the primate spatial cognitive system directly motivate properties of spatial language, giving rise to strong universals (such as the closed class/open class distinction) and constraints on typological variation. Clearly, such claims need to be evaluated against comprehensive linguistic data. The semantic and cognitive domain of space has been paradigmatic in cognitive typology. One aspect of language variation that has been subject to extensive cross-linguistic study from a cognitive perspective recently is motion-event typology, i.e. the way different languages frame events of translocation. Our research will deepen our existing analyses focussing on Amondawa (Sampaio et al, in press) and Thai. The spatial domain has also been adduced in support of strong claims for linguistic and cognitive universals. There has been much research on such hypothesised universals in metaphorical mapping from the conceptual domain of space onto conceptual domains that are less accessible to experience; however, details of that mapping vary considerably. Specifically, recent research suggests that the cultural conventions entrenched in a particular language might be more important than previously thought. Our research extends the database to allow a comprehensive understanding of sign use in spatial conceptualisation and metaphor.

\subsection{Imitation and mimesis}

Within the chain of the usually recognised stages from ritualised movements, imperative pointing to declarative pointing, the relationship between expression and content becomes sufficiently distinct to allow the emergence of the sign function. However, imperative pointing can be shown to arise from ritualisation, while (human) declarative pointing emerges by imitation (Brinck, 2003). It has also not been sufficiently well explained how the ability to imitate gestures and use them in intentional communication relates to action understanding and cooperation (Brinck \& Gärdenfors, 2003). We hold that the concept of bodily 
mimesis (Donald 1991) can help us reach a better understanding of these stages in the use of gesture. We distinguish between a dyadic form of mimesis, the clearest form of which is imitation, and triadic mimesis, where someone mimes something for someone else, e.g. pantomime (Zlatev, Persson \& Gärdenfors, 2005). Research has shown that apes, especially those raised and trained by humans, are capable of mimesis in its dyadic form (Call, 2001). In contrast, it does not seem that apes are also capable of triadic mimesis in the form of iconic gestures or declarative pointing (Tomasello et al, 1997) but there is some evidence to the contrary. We are currently investigating the basis for the differences in the mimetic skills of apes and humans. In particular, we are focusing on the ability to use imitation to acquire novel communicative signs.

Furthermore, we are investigating whether other mechanisms than imitation could be involved in the rise of the first communicative gestures of prelinguistic children. One possibility is that children could create novel representational acts on the basis of the similarity of the observed objects or events, i.e. on the basis of primary iconicity (see 2.2 above). Evidence for this would be if children from (widely) different linguistic and cultural environments have similar gestures. To study the role of cultural transmission for the emergence of children's gestures we are comparing longitudinal data consisting of spontaneous videotaped interactions between caregivers and children from Thailand and Sweden.

\subsection{Intersubjectivity and conventions}

The goal in this domain is to define the progressive emergence of intersubjectivity in evolution and ontogeny as well as to study the role of culture-specific patterns for the formation of conventions. The two are intimately related since intersubjectivity involves the ability to share the mentality of others and conventions exist as a form of shared, common knowledge. A basic form of intersubjectivity involves the awareness of others' feelings and attention to oneself; this requires both a species-general capacity for empathy (Preston \& de Waal, 2002), but also engagement in acts of mutual attention, displayed in phenomena such as eye-contact, intense smiling, coyness, calling vocalizations and showing-off (Reddy, 1991). Careful comparisons of videotaped episodes of mother-infant interactions in humans and non-human apes will show to what extent such behaviours are specific for our species. A second developmental and possibly evolutionary stage of intersubjectivity involves the ability to understand the intentions of others. Children master this second stage around the age of one, and newer evidence and analyses show that chimpanzees too achieve this level (Hare, Call \& Tomasello, 2001), at least in competitive contexts. A third stage involves understanding others' attention to one's own attention and communicative intentions. It has been suggested that apes cannot master this in cooperative settings, but this has not been explored in 
the context of mother-infant interaction. Experiments with food sharing between ape mothers and infants, in various contexts, are being conducted in order to test their potentials for collaboration and gestural communication.

Understanding the relationship between sign use and intersubjectivity is further enhanced by a cross-cultural investigation of the framing of compliance in early parent-infant interactions in two different cultural environments (Portsmouth, UK and Hyderabad, India). Compliance, considered a sign of developmental and interpersonal maturity by Western psychology, is in fact an intrinsically relational and culturally variable achievement. For infants to become aware that they may need to amend their own actions in relation to others' intentions, they not only need a certain level of developmental maturity, but an environment where others are in fact communicating such intentions. This requires not only a belief in the desirability of compliance but also in its possibility, beliefs which vary between different situations and cultures. The "Western" focus on consistency in parental actions and on the positive correlates of child compliance neglects the complexity of communication in such engagements, particularly in Asian cultures where negotiation tends to predominate over rules even in childhood (Reddy, 1983). We use parental recognition, emphasis and negotiation of different situations as a frame for the understanding of intentions and the emergence of sign use.

\section{Conclusions}

The investigations in the different social-cognitive domains described in this article are being conducted in parallel, with extensive sharing of methodologies and results. Since we hold that each domain plays a key role in providing cognitive prerequisites for the development of sign use, and at the same time is transformed by the acquisition of the latter, we expect to find considerable similarities and interactions between developments in the domains. Finally, we plan to integrate all the results in a coherent theory of semiotic development in which we (a) identify stage-like transitions within each one of the five socialcognitive domains, (b) investigate interactions, dependencies and synergies between such transitions across the different cognitive domains and (c) relate such transitions to sign use, both in terms of precursors and prerequisites and in terms of the transformations wrought in the domains by the acquisition and development of semiotic skills. Our contention is that such a theory is hitherto lacking. Even though the SEDSU project is only 9 months old, we are confident that due to its interdisciplinary, integrative character it will at least contribute to such a theory, and hence, to explaining the evolution of language. 


\section{References}

Barbet, I. \& Fagot, J. (2002). Perception of the corridor illusion by baboons. Behavioural Brain Research, 132, 111-115.

Brinck, I. (2003). The pragmatics of imperative and declarative pointing, Cognitive Science Quarterly, 3(4), 2003, 429-446.

Brinck, I. \& Gärdenfors, P. (2003). Co-operation and communication in apes and humans", Mind and Language, 18(5), 484-501.

Call, J. (2001). Body imitation in an enculturated orangutan (Pongo pygmaeus). Cybernetics and Systems, 32(1-2), 97-119.

Christiansen, M. H. \& Kirby S., (2003). (Eds.) Language evolution. Oxford: Oxford University Press.

Davidoff, J., Davies, I. \& Roberson, D. (1999). Colour categories of a stone-age tribe. Nature, 398, 203-204.

Donald, M. (1991). Origins of the modern mind. Three stages in the evolution of culture and cognition, Cambridge, Mass.: Harvard University Press.

Fagot, J. \& Tomonaga, M. (1999). Comparative assessment of global-local processing in humans (Homo sapiens) and chimpanzees (Pan troglodytes): Use of a visual search task with compound stimuli. Journal of Comp. Psychology, 113, 3-12.

Fagot, J., Goldstein, J., Davidoff, J. \& Pickering, A. (in press). Cross species differences in colour categorisation. Psychonomic bulletin and review.

Gibson, J. (1982). Reasons for realism. Selected essays of James J. Gibson, E. Reed \& R. Jones (Eds.). Hillsdale, NJ: Lawrence Erlbaum.

Hare, B., Call, J., \& Tomasello, M., (2001). Do chimpanzees know what conspecifics know and do not know? Animal Behaviour, 61, 139-151.

Heaton (in press). Interval and contour processing in autism. Journal of Autism and Developmental Disorders.

Peirce, C. S. (1931-58). Collected Papers I-VIII. Hartshorne, C, Weiss, P, \& Burks, A, (Eds.). Cambridge, MA: Harvard University Press.

Piaget, J. (1945). La formation du symbole chez l'enfant. Neuchatel: Delachaux \& Niestlé. Third edition 1967.

Potì, P., Bartolommei, P. \& Saporiti, M. (2005). Landmark use by Cebus apella. International Journal of Primatology, 26 (4), 921-948.

Preston, S. D. and de Waal, F. B. M. (2002). Empathy: its ultimate and proximal causes. Behavioral and Brain Sciences, 25, 1-20.

Reddy, V. (1983). Responsiveness and rules: Parent-child interaction in Scotland and India. Unpublished PhD Thesis, University of Edinburgh.

Reddy, V. (1991). Teasing, joking and mucking about in the first year. In A. Whiten (Ed.) Natural theories of mind (pp. 143-158). Oxford: Blackwell.

Sampaio, W., Sinha, C. and da Silva Sinha, W. (in press) Mixing and mapping: motion and manner in Amondawa. In E. Lieven (Ed.) Crosslinguistic Approaches to the Psychology of Language: Research in the Tradition of 
Dan Slobin. Mahwah, NJ: Lawrence Earlbaum Associates.

Sonesson, G. (2000). Iconicity in the ecology of semiosis. In T. D. Johansson, M. Skov \& B. Brogaard (Eds.) Iconicity - a fundamental problem in semiotics, (pp 59-80). Aarhus: NSU Press.

Spinozzi G., De Lillo C., \& Truppa V. (2003). Global and local processing of hierarchical visual stimuli in tufted capuchin monkeys (Cebus apella). Journal of Comparative Psychology, 117, 15-23.

Tomasello, M. (1999). The cultural origins of human cognition. Cambridge, MA: Harvard University Press.

Tomasello, M., Call, J., Warren, J., Frost, G. T., Carpenter, M., \& Nagell, K. (1997). The ontogeny of chimpanzee gestural signals: A comparison across groups and generations. Evolution of Communication, 1, 223-259.

Zlatev, J., Persson, T. \& Gärdenfors, P. (2005). Bodily mimesis as the "missing link” in human cognitive evolution. LUCS 121. Lund: Lund University. 